\title{
TMD Evolution at Moderate Hard Scales
}

\section{Ted C. Rogers*}

Department of Physics, Old Dominion University, Norfolk, VA 23529, USA

and Theory Center, Jefferson Lab, 12000 Jefferson Avenue, Newport News, VA 23606, USA

E-mail: trogers@odu.edu

\section{John Collins}

104 Davey Lab., Penn State University, University Park PA 16802, USA

E-mail: jcc8@psu.edu

We summarize some of our recent work on non-perturbative transverse momentum dependent (TMD) evolution, emphasizing aspects that are necessary for dealing with moderately low scale processes like semi-inclusive deep inelastic scattering.

PoS.cls, January 11, 2016, JLAB-THY-16-2196, DOE/OR/23177-3644

QCD Evolution 2015 -QCDEV2015-

26-30 May 2015

Jefferson Lab (JLAB), Newport News Virginia, USA

${ }^{*}$ Speaker. 


\section{TMD factorization and non-perturbative evolution}

The purpose of this talk is to summarize results recently presented in Ref. [1]. We will discuss the Collins-Soper-Sterman (CSS) form of TMD factorization in the updated version presented in Ref. [2]. (See Ref. [3] for a general overview and for references.) For these proceedings, the relevant aspects of the TMD factorization theorems are the following:

- The unpolarized cross section for a process like Drell-Yan scattering is expressible as

$$
\begin{aligned}
& \frac{\mathrm{d} \sigma}{\mathrm{d}^{4} q \mathrm{~d} \Omega} \\
& =\frac{2}{s} \sum_{j} \frac{\mathrm{d} \hat{\sigma}_{j \bar{J}}\left(Q, \mu \rightarrow Q ; \alpha_{S}(Q)\right)}{\mathrm{d} \Omega} \int \mathrm{d}^{2} \mathbf{b} e^{i \mathbf{q}_{\mathrm{T}} \cdot \mathbf{b}} \tilde{F}_{j / A}\left(x_{A}, \mathbf{b} ; Q^{2}, Q\right) \tilde{F}_{\overline{\mathrm{J}} / B}\left(x_{B}, \mathbf{b} ; Q^{2}, Q\right) \\
& +\operatorname{large} q_{\mathrm{T}} \text { "Y-term" correction. }
\end{aligned}
$$

where $\mathrm{d} \hat{\sigma}_{j \bar{J}} / \mathrm{d} \Omega$ is a hard partonic cross section and $\tilde{F}\left(x, \mathbf{b} ; Q^{2}, Q\right)$ is a TMD parton distribution function (PDFs) in coordinate space evaluated with a hard scale $Q$.

- Collins-Soper (CS) evolution applied to an individual TMD PDF leads to

$$
\frac{\partial}{\partial \ln Q} \ln \tilde{F}_{j / A}\left(x_{A}, \mathbf{b}_{\mathrm{T}} ; Q^{2}, Q\right)=\tilde{K}\left(b_{\mathrm{T}} ; Q\right)+b_{\mathrm{T}} \text { Independent Terms }
$$

The " $b_{\mathrm{T}}$ Independent Terms" only affect the normalization of $\tilde{F}$ but not its shape.

- The kernel $\tilde{K}\left(b_{\mathrm{T}} ; Q\right)$ is is strongly universal. At small $b_{\mathrm{T}}$ its $b_{\mathrm{T}}$-dependence is perturbatively calculable with $1 / b_{\mathrm{T}}$ acting as a hard scale. At large $b_{\mathrm{T}}$ its $b_{\mathrm{T}}$-dependence is nonperturbative.

- For all $b_{\mathrm{T}}, \tilde{K}\left(b_{\mathrm{T}} ; Q\right)$ obeys the renormalization group (RG) equation:

$$
\frac{\mathrm{d}}{\mathrm{d} \ln \mu} \tilde{K}\left(b_{\mathrm{T}} ; \mu\right)=-\gamma_{K}\left(\alpha_{s}(\mu)\right) .
$$

At small $b_{\mathrm{T}}$, one hopes to exploit perturbation theory with $1 / b_{\mathrm{T}}$ as a hard scale to calculate $\tilde{K}\left(b_{\mathrm{T}} ; Q\right)$ while at large $b_{\mathrm{T}}$ a non-perturbative parametrization is needed. In the non-perturbative region, one hopes to exploit the strong universality of $\tilde{K}\left(b_{T} ; Q\right)$ to make predictions. One needs a prescription to demarcate what constitutes large and small $b_{\mathrm{T}}$. To smoothly interpolate between the two regions, one imposes a gentle cutoff on large $b_{\mathrm{T}}$. A common choice of cutoff function is

$$
\mathbf{b}_{*}\left(\mathbf{b}_{\mathrm{T}}\right)=\frac{\mathbf{b}_{\mathrm{T}}}{\sqrt{1+b_{\mathrm{T}}^{2} / b_{\max }^{2}}} .
$$

Then an RG scale defined as $\mu_{b_{*}} \equiv C_{1} / b_{*}$ approaches $C_{1} / b_{\mathrm{T}}$ at small $b_{\mathrm{T}}$ and $C_{1} / b_{\max }$ at large $b_{\mathrm{T}}$. We can separate $\tilde{K}\left(b_{\mathrm{T}} ; Q\right)$ into a large $b_{\mathrm{T}}$ part and a small $b_{\mathrm{T}}$ part by adding and subtracting $\tilde{K}\left(b_{*} ; Q\right)$ in Eq. (1.2):

$$
\frac{\partial}{\partial \ln Q} \ln \tilde{F}_{j / A}\left(x_{A}, \mathbf{b}_{\mathrm{T}} ; Q^{2}, Q\right)=\tilde{K}\left(b_{*} ; Q\right)+\left[\tilde{K}\left(b_{\mathrm{T}} ; Q\right)-\tilde{K}\left(b_{*} ; Q\right)\right]+b_{\mathrm{T}} \text { Independent Terms } .
$$


The $g_{K}\left(b_{\mathrm{T}} ; b_{\max }\right)$ function is defined as the term $\tilde{K}\left(b_{\mathrm{T}} ; Q\right)-\tilde{K}\left(b_{*} ; Q\right)$, so that

$$
\frac{\partial}{\partial \ln Q} \ln \tilde{F}_{j / A}\left(x_{A}, \mathbf{b}_{\mathrm{T}} ; Q^{2}, Q\right)=\tilde{K}\left(b_{*} ; Q\right)-g_{K}\left(b_{\mathrm{T}} ; b_{\max }\right)+b_{\mathrm{T}} \text { Independent Terms } .
$$

By definition, the right side of Eq. (1.6) is exactly independent of $b_{\max }$. From Eq. (1.3), $g_{K}\left(b_{\mathrm{T}} ; b_{\max }\right)$ is also exactly independent of $Q$. The $Q$ dependence in each of the terms in the definition of $g_{K}\left(b_{\mathrm{T}} ; b_{\max }\right)$ cancels. We can apply Eq. (1.3) to exploit RG improvement in the calculation of $\tilde{K}\left(b_{*} ; Q\right)$ :

$$
\tilde{K}\left(b_{*} ; Q\right)=\tilde{K}\left(b_{*} ; \mu_{b_{*}}\right)-\int_{\mu_{b_{*}}}^{Q} \frac{\mathrm{d} \mu^{\prime}}{\mu^{\prime}} \gamma_{K}\left(\alpha_{s}\left(\mu^{\prime}\right)\right) .
$$

So, the evolution of the shape of $\tilde{F}_{j / A}\left(x_{A}, \mathbf{b}_{\mathrm{T}} ; Q^{2}, Q\right)$ is is given by

$$
\begin{aligned}
& \frac{\partial}{\partial \ln Q} \ln \tilde{F}_{j / A}\left(x_{A}, \mathbf{b}_{\mathrm{T}} ; Q^{2}, Q\right) \\
& =\tilde{K}\left(b_{*} ; \mu_{b_{*}}\right)-\int_{\mu_{b_{*}}}^{Q} \frac{\mathrm{d} \mu^{\prime}}{\mu^{\prime}} \gamma_{K}\left(\alpha_{s}\left(\mu^{\prime}\right)\right)-g_{K}\left(b_{\mathrm{T}} ; b_{\max }\right)+b_{\mathrm{T}} \text { Independent Terms. }
\end{aligned}
$$

The partial derivative symbol means $x_{A}$ is to be held fixed. The $g_{K}\left(b_{T} ; b_{\max }\right)$ function inherits the universality properties of $\tilde{K}\left(b_{\mathrm{T}} ; \mu\right)$. In particular, it is related to the vacuum expectation value of a relatively simple Wilson loop. It is independent of any details of the process and is even the same if the $\operatorname{PDF} \tilde{F}_{j / A}\left(x_{A}, \mathbf{b}_{\mathrm{T}} ; Q^{2}, Q\right)$ is replaced with a fragmentation function. Thus we say that $g_{K}\left(b_{\mathrm{T}} ; b_{\max }\right)$ is "strongly" universal; see the graphic in Fig. 1 . The $g_{K}\left(b_{\mathrm{T}} ; b_{\max }\right)$ function is often called the "non-perturbative" part of the evolution since it can contain non-perturbative elements. This is a slight misnomer, however, since $g_{K}\left(b_{\mathrm{T}} ; b_{\max }\right)$ can contain perturbative contributions as well. Indeed, at very small $b_{\mathrm{T}}$ it is entirely perturbatively calculable, though suppressed by powers of $b_{\mathrm{T}} / b_{\max }$, according to its definition in Eq. (1.5).

\section{Large $b_{\mathbf{T}}$ behavior}

A common choice for non-perturbative parametrizations of $g_{K}\left(b_{\mathrm{T}} ; b_{\max }\right)$ is a power-law form. These tend to yield reasonable success in fits that involve at least moderately high scales $Q$ [4]. However, extrapolations of those fits to lower values of $Q$ (such as those corresponding to many current SIDIS experiments) appear to appear to produce evolution that is far too rapid [5, 6]. In this talk, we carefully examine the underlying physics issues surrounding non-perturbative evolution and, on the basis of those considerations, we will propose a form for $g_{K}\left(b_{\mathrm{T}} ; b_{\max }\right)$ that accommodates both large and small $Q$ behavior.

We will first write down our proposed ansatz for $g_{K}\left(b_{T} ; b_{\max }\right)$ and then spend the remainder of the talk discussing its justifications. Our proposal is

$$
g_{K}\left(b_{\mathrm{T}} ; b_{\max }\right)=g_{0}\left(b_{\max }\right)\left(1-\exp \left[-\frac{C_{F} \alpha_{s}\left(\mu_{b_{*}}\right) b_{\mathrm{T}}^{2}}{\pi g_{0}\left(b_{\max }\right) b_{\max }^{2}}\right]\right),
$$

where

$$
g_{0}\left(b_{\max }\right)=g_{0}\left(b_{\max , 0}\right)+\frac{2 C_{F}}{\pi} \int_{C_{1} / b_{\max , 0}}^{C_{1} / b_{\max }} \frac{\mathrm{d} \mu^{\prime}}{\mu^{\prime}} \alpha_{s}\left(\mu^{\prime}\right) .
$$




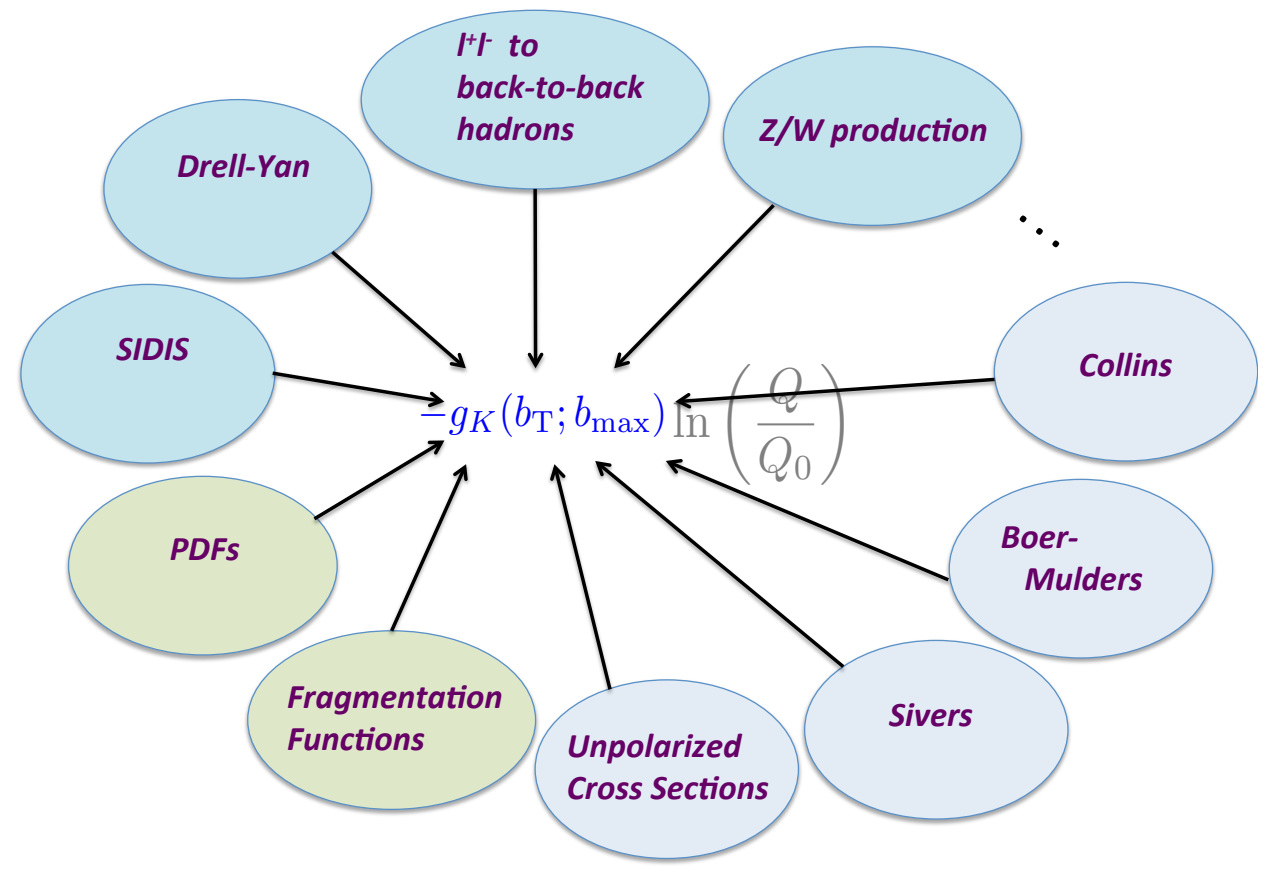

Figure 1: Strong universality of the non-perturbative evolution parametrized by $g_{K}\left(b_{\mathrm{T}} ; b_{\max }\right)$. The $-g_{K}\left(b_{\mathrm{T}} ; b_{\max }\right) \ln \left(Q / Q_{0}\right)$ combination appears exponentiated in the evolved cross section expression.

The only parameter of the model is $g_{0}\left(b_{\max }\right)$ and it varies with $b_{\max }$ according to Eq. (2.2). $b_{\max , 0}$ is a boundary value for $g_{0}$ relative to which other values are determined.

First, note that the a small $b_{\mathrm{T}} / b_{\max }$ expansion of Eq. (2.1) gives

$$
g_{K}\left(b_{\mathrm{T}} ; b_{\max }\right)=\frac{C_{F}}{\pi} \frac{b_{\mathrm{T}}^{2}}{b_{\max }^{2}} \alpha_{s}\left(\mu_{b_{*}}\right)+O\left(\frac{b_{\mathrm{T}}^{4} C_{F}^{2} \alpha_{s}\left(\mu_{b_{*}}\right)^{2}}{b_{\max }^{4} \pi^{2} g_{0}\left(b_{\max }\right)}\right)
$$

while an expansion of the exact definition of $-g_{K}\left(b_{\mathrm{T}} ; b_{\max }\right)$ in Eq. (1.5) is

$$
\begin{aligned}
g_{K}\left(b_{\mathrm{T}} ; b_{\max }\right) & =-\tilde{K}\left(b_{\mathrm{T}} ; \mu_{b_{*}} ; \alpha_{s}\left(\mu_{b_{*}}\right)\right)+\tilde{K}\left(b_{*} ; \mu_{b_{*}} ; \alpha_{s}\left(\mu_{b_{*}}\right)\right) \\
& =\frac{C_{F}}{\pi} \frac{b_{\mathrm{T}}^{2}}{b_{\max }^{2}} \alpha_{s}\left(\mu_{b_{*}}\right)+O\left(\frac{b_{\mathrm{T}}^{4}}{\pi^{2} b_{\max }^{4}} \alpha_{s}\left(\mu_{b_{*}}\right)^{2}\right)
\end{aligned}
$$

So, the exact definition and Eq. (2.1) match in the small $b_{\mathrm{T}}$ limit.

\section{Conditions on $g_{K}\left(b_{\mathbf{T}} ; b_{\max }\right)$}

Our description of the large $b_{\mathrm{T}}$ limit of correlation functions like $\tilde{F}\left(x_{A}, \mathbf{b}_{\mathrm{T}} ; Q^{2}, Q\right)$ is motivated by the general observation that the analytic properties of correlation functions imply an exponential 
coordinate dependence, with a possible power-law fall-off, for the large $b_{\mathrm{T}}$ limit. That is, neglecting perturbative contributions,

$$
\tilde{F}\left(x_{A}, \mathbf{b}_{\mathrm{T}} ; Q^{2}, Q\right) \stackrel{b_{\mathrm{T}} \rightarrow \infty}{\sim} \frac{1}{b_{\mathrm{T}}^{\alpha}} e^{-m b_{\mathrm{T}}},
$$

with $m$ and $\alpha$ independent of $Q$. See, for example, Ref. [7]. Therefore, from Eq. (1.2), $\tilde{K}\left(b_{\mathrm{T}} ; Q\right)$ must approach a $b_{\mathrm{T}}$-independent constant at large $b_{\mathrm{T}}$.

The set of requirements on $g_{K}\left(b_{\mathrm{T}} ; b_{\max }\right)$ is

1. $\tilde{K}\left(b_{\mathrm{T}} ; \mu_{b_{*}}\right) \stackrel{b_{\mathrm{T}} \rightarrow 0}{=} \tilde{K}\left(b_{\mathrm{T}} ; C_{1} / b_{\mathrm{T}}\right)$ is calculable entirely in perturbation theory with $C_{1} / b_{\mathrm{T}}$ playing the role of a hard scale.

2. $\tilde{K}\left(b_{\mathrm{T}} ; Q\right)$ approaches a constant at $b_{\mathrm{T}} / b_{\max } \rightarrow \infty$. The constant can be $Q$-dependent, but the $Q$-dependence can be calculated perturbatively for all $b_{\mathrm{T}}$ from Eq. (1.3).

3. Because of item $2, g_{K}\left(b_{\mathrm{T}} ; b_{\max }\right)$ must approach a constant at large $b_{\mathrm{T}}$, but the constant depends on $b_{\max }$.

4. At small $b_{\mathrm{T}}, g_{K}\left(b_{\mathrm{T}} ; b_{\max }\right)$ is a power series in $\left(b_{\mathrm{T}} / b_{\max }\right)^{2}$ with perturbatively calculable coefficients, as in Eqs. $(2.3,2.4)$.

5. By definition, the right side of Eq. (1.8) is independent of $b_{\max }$ and this should be preserved as much as possible in the functional form that parametrizes $g_{K}\left(b_{\mathrm{T}} ; b_{\max }\right)$. For small $b_{\mathrm{T}}$, this means

$$
\left.\underset{b_{\mathrm{T}} \ll b_{\max }}{\operatorname{asy}} \frac{\mathrm{d}}{\mathrm{d} b_{\max }} g_{K}\left(b_{\mathrm{T}} ; b_{\max }\right)\right|_{\text {parametrized }}=\left.\underset{b_{\mathrm{T}} \ll b_{\max }}{\operatorname{asy}} \frac{\mathrm{d}}{\mathrm{d} b_{\max }} g_{K}\left(b_{\mathrm{T}} ; b_{\max }\right)\right|_{\text {truncated PT }}
$$

where "parametrized" refers to a specific model of $g_{K}\left(b_{\mathrm{T}} ; b_{\mathrm{max}}\right)$ while "truncated PT" refers to a truncated perturbative expansion. Eqs. $(2.3,2.4)$ satisfy this requirement through order $\alpha_{s}\left(\mu_{b_{*}}\right)$.

6. At large $b_{\mathrm{T}}, b_{\max }$-independence of the exact $\tilde{K}\left(b_{\mathrm{T}}, \mu\right)$ implies that, to a useful approximation,

$$
\frac{\mathrm{d}}{\mathrm{d} \ln b_{\max }} g_{K}\left(b_{\mathrm{T}}=\infty ; b_{\max }\right)=\left[\frac{\mathrm{d} \tilde{K}\left(b_{\max } ; C_{1} / b_{\max }\right)}{\mathrm{d} \ln b_{\max }}-\gamma_{K}\left(\alpha_{s}\left(C_{1} / b_{\max }\right)\right)\right]_{\text {truncated PT }},
$$

as obtained from Eq. (1.7) and the definition of $g_{K}$. Equation (2.2) ensures that Eq. (2.1) satisfies Eq. (1.8) so long as everything is calculated only to order $\alpha_{s}\left(\mu_{b_{*}}\right)$. Enforcing both Eq. (3.2) and Eq. (3.3) simultaneously means $g_{K}\left(b_{\mathrm{T}} ; b_{\max }\right)$ will produce a $b_{\max }$ independent contribution to $\tilde{K}\left(b_{\mathrm{T}} ; Q\right)$ for all $b_{\mathrm{T}}$ except perhaps for an intermediate region at the border between perturbative and non-perturbative $b_{\mathrm{T}}$-dependence. The residual $b_{\max }$ dependence there can be reduced by calculating higher orders and refining knowledge of non-perturbative behavior.

For a much more detailed discussion of these considerations, see Sect. VII of Ref. [1]. Equation (2.1) is one of the simplest models that satisfies all 6 of these properties simultaneously. 


\section{Conclusion}

In Sect. 3 we enumerated properties that a model of $g_{K}\left(b_{\mathrm{T}} ; b_{\max }\right)$ needs tp ensure basic consistency in a calculation $\tilde{K}\left(b_{\mathrm{T}} ; Q\right)$. A simple parametrization was proposed in Sect. 2.

Note that a quadratic $\left(b_{\mathrm{T}} / b_{\max }\right)^{2}$ dependence at small $b_{\mathrm{T}}$ emerges naturally from (2.1), but with a perturbatively calculable coefficient. Furthermore, the dependence is not exactly quadratic because the coefficients contain logarithmic $b_{\mathrm{T}}$ dependence through $\alpha_{s}\left(\mu_{b_{*}}\right)$.

In a process dominated by very large $b_{\mathrm{T}}$, Sect. 3 and Eq. (2.1) predict an especially simple evolution for the low- $Q$ cross section. Namely, the cross section scales as $\left(Q / Q_{0}\right)^{a}$ where $a$ is combination of $g_{K}\left(\infty, b_{\max }\right)$ and perturbatively calculable quantities. (See Eq. $(85,86)$ of Ref. [1].)

Future phenomenological work should include efforts to constrain $g_{0}$. Because of its strongly universal nature, this offers a relatively simple way to test TMD factorization.

\section{Acknowledgments}

This work was supported by DOE contract No. DE-AC05-06OR23177, under which Jefferson Science Associates, LLC operates Jefferson Lab., and by DOE grant No. DE-SC0013699.

\section{References}

[1] J. Collins and T. Rogers, "Understanding the large-distance behavior of transverse-momentum-dependent parton densities and the Collins-Soper evolution kernel," Phys. Rev. D 91, no. 7, 074020 (2015) doi:10.1103/PhysRevD.91.074020 [arXiv:1412.3820 [hep-ph]].

[2] J. Collins, "Foundations of perturbative QCD," (Cambridge monographs on particle physics, nuclear physics and cosmology. 32)

[3] T. C. Rogers, "An Overview of Transverse Momentum Dependent Factorization and Evolution," arXiv:1509.04766 [hep-ph].

[4] A. V. Konychev and P. M. Nadolsky, "Universality of the Collins-Soper-Sterman nonperturbative function in gauge boson production," Phys. Lett. B 633, 710 (2006) doi:10.1016/j.physletb.2005.12.063 [hep-ph/0506225].

[5] P. Sun and F. Yuan, "Transverse momentum dependent evolution: Matching semi-inclusive deep inelastic scattering processes to Drell-Yan and W/Z boson production," Phys. Rev. D 88, no. 11, 114012 (2013) doi:10.1103/PhysRevD.88.114012 [arXiv:1308.5003 [hep-ph]].

[6] C. A. Aidala, B. Field, L. P. Gamberg and T. C. Rogers, "Limits on transverse momentum dependent evolution from semi-inclusive deep inelastic scattering at moderate $Q$," Phys. Rev. D 89, no. 9, 094002 (2014) doi:10.1103/PhysRevD.89.094002 [arXiv:1401.2654 [hep-ph]].

[7] P. Schweitzer, M. Strikman and C. Weiss, "Intrinsic transverse momentum and parton correlations from dynamical chiral symmetry breaking," JHEP 1301, 163 (2013) doi:10.1007/JHEP01(2013)163 [arXiv:1210.1267 [hep-ph]]. 